\title{
Plasmonic Amplifier of the Evanescent Field of Free Electrons
}

\author{
Jin-Kyu So1, Jun-Yu Ou1, Giorgio Adamo',2, F. Javier García de Abajo1,3, Kevin F. MacDonald1, \\ and Nikolay I. Zheludev ${ }^{1,2}$ \\ 1. Optoelectronics Research Centre \& Centre for Photonic Metamaterials, University of Southampton, SO17 1BJ, UK \\ 2. Centre for disruptive Photonic Technologies, Nanyanag Technological University, Singapore \\ 3. IQFR - CSIC, Serrano 191, 28006 Madrid, Spain
}

\begin{abstract}
We show experimentally for the first time that free-electron evanescent fields can be amplified by a plasmonic nanolayer in much that same way as optical evanescent fields are amplified in the poor-man's superlens.

The manipulation of evanescent electromagnetic fields has been a key motivation in the field of plasmonics. However, one class of evanescent field has been excluded from consideration here - the evanescent field of moving free electrons. The electromagnetic energy of free electrons moving in vacuum exists in the form of evanescent waves and it can be out-coupled to light only when the moving electrons are in close proximity to a 'slow-wave medium' or optical inhomogeneity. The former gives rise to the well-known Cerenkov radiation, the latter to diffraction or Smith-Purcell radiation. Here, in analogy to the 'poor-man's superlens', wherein the evanescent field component of light from an object is restored by a thin silver layer to beat the diffraction limit [1], we demonstrate the use of a thin silver layer to amplify the free-electron evanescent field before it is outcoupled into light by a nano-grating. The silver layer supports the phase-velocity matched surface plasmons, and it leads to the amplification of the electrons' evanescent field.
\end{abstract}
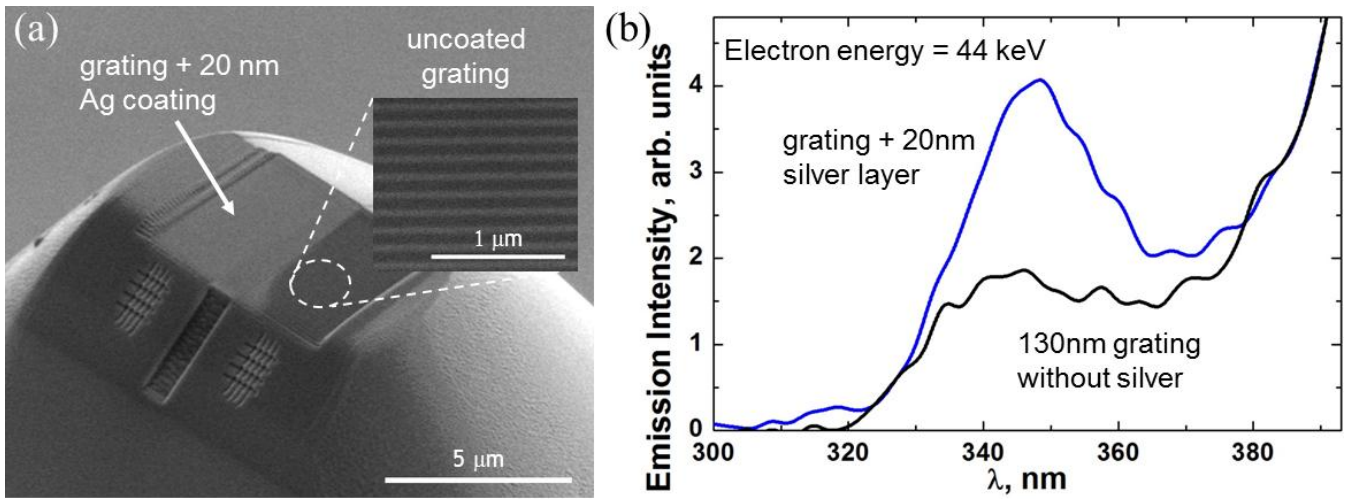

Fig. 1 (a) Electron microscope image of a tapered multi-mode optical fibre end face with a planarized, $130 \mathrm{~nm}$ period aluminium grating partly coated with a $20 \mathrm{~nm}$ layer of silver. (b) Electron-beam-driven light emission spectra from gratings with (blue) and without (black) the silver film.

A grating period is chosen such that the phase-velocity matched fields can be out-coupled along the surface-normal direction - a condition satisfied by a $130 \mathrm{~nm}$ period for an electron energy of $40 \mathrm{keV}$. In order to put a flat silver layer, planarized nano-gratings are manufactured on the end faces of tapered optical fibres by focused ion-beam milling and part-coated with a $20 \mathrm{~nm}$ thick silver layer as shown in Fig. 1 (a). The fibre face is aligned parallel to the electron beam trajectory in a scanning electron microscope (SEM) and the generated light is collected via the hosting fibre for spectroscopic analysis.

The effect of an intermediate silver film is examined by comparing peak emission wavelengths and intensities from coated and uncoated nano-gratings at electron energies between 40 and $50 \mathrm{keV}$. The silver layer enhances emission intensity by a factor of up to 2.8 at $44 \mathrm{keV}$, while also red-shifting the emission, indicating strong coupling of the electron evanescent field to surface plasmons on the silver (see Fig. 1 (b)).

The concept of enhancing the free-electron evanescent field by introducing an intermediate layer of plasmonic metal can readily be applied to any part of the electromagnetic spectrum by suitably engineering the nanostructure. Furthermore, the configuration pioneered in this experiment could serve as a platform for investigating proximity interactions between free electrons and a wide range of nanophotonic structures.

\section{References}

[1] J. B. Pendry, "Negative Refraction Makes a Perfect Lens," Phys. Rev. Lett. 85, 3966 (2000). 